\title{
CHARACTERIZATION OF DIFFUSIONNAL TRANSFERS OF BOUND WATER AND WATER VAPOR IN BEECH AND SPRUCE
}

\author{
N. Mouchot, F. Thiercelin, P. Perre, A. Zoulalian*
}

\begin{abstract}
In the hygroscopic range of wood and without any thermal gradient, transfer of water vapor and bound water is carried out by diffusion. In this study, we experimentally determined the diffusivity values for vapor and bound water in the three orthotropic directions of wood for two species (beech and spruce). We then evaluated in steady-state the fluxes for the two forms of water and showed the influence of wood morphologies and transfer direction on these fluxes.
\end{abstract}

Keywords: Diffusivity, Water vapor, Bound water, Beech, Spruce

\section{INTRODUCTION}

At low temperature and in the hygroscopic range of wood (moisture content lower than the fiber saturation point), the transfer of water existing in the form of water vapor and bound (or adsorbed) water is mainly done by diffusion. The convective fluxes due to total pressure differences are generally negligible in the absence of a temperature gradient. In the presence of a moisture gradient, the water transfer is carried out by diffusion in the aforementioned two forms. We have shown (Mouchot and Zoulalian 2003) that it is possible to experimentally attain in an indirect way the apparent diffusivity of water vapor and thereafter, in a direct way the apparent diffusivity of bound water. In steady-state, it is consistent to admit that locally water vapor and bound water are in thermodynamic equilibrium. However wood, whatever the species are, being anisotropic, the diffusivities in the three orthotropic directions are different because they depend on the morphology of the species. The fluxes of diffusive transfers are consequently dissimilar, for both vapor and bound water. In a given direction, the comparison between these two fluxes allows to specify the predominant transfer mechanism.

The aim of this publication is a quantification of diffusive transfers of water vapor and bound water for samples of two local species (beech and spruce) in the three orthotropic directions.

Before presenting and discussing our obtained results, we remind briefly the experimental methodology employed to reach the apparent diffusivities of water vapor and bound water in a wood

\footnotetext{
LERMAB - UMR INRA - ENGREF - UHP n;1093 - Faculté des Sciences et Techniques - Université Henri Poincaré Nancy 1 - BP 239 - 54506 Vandoeuvre-lès-Nancy 
sample. For more details on the methodology, the Proceedings of the sixth French-Canadian Symposium could be consulted (Mouchot and Zoulalian 2003).

\section{MATERIALS AND METHODS}

The measurements were done on beech (Fatus Sylvatica) and spruce ( Picea Sp. )samples in the form of circular disks with a diameter close to $70 \mathrm{~mm}$ and with variable thicknesses in the diffusion transfer direction between 9 and $30 \mathrm{~mm}$ (Table 1).

The values of the diffusivities were obtained by an indirect way; we determined first the apparent diffusivity of a non- adsorbent gaseous solute. The measurements were done in a Wicke and Kallenbach diffusion cell (Figure 1), made up of two compartments between which is the wood sample. The cell can work in batch or open system, depending on the value of the transferred flow in comparison with the flow supplying the two compartments. In an open system the two cells are fed simultaneously by two different gas flows (nitrogen and helium). More precisely, if the transfer flow is low, the cell runs in batch system. On the other hand, if the magnitude of the transferred flow is similar to the supplying flows, the cell runs in open system. For beech and spruce samples, the determination of the diffusivity in the axial direction is made in open system. On the other hand, the evolution of the cell in the batch system is necessary for the determination of the diffusivities in the radial and tangential directions. The diffusivities of the non-adsorbent solute are obtained by using the binary system nitrogen-helium, the gaseous solute analyzed being helium.

Table 1: Geometrical characteristics of the studied samples

\begin{tabular}{|c|c|c|c|c|c|}
\hline Samples & $\begin{array}{c}\text { Direction of } \\
\text { the transfer }\end{array}$ & Species & $\mathbf{S}\left(\mathrm{mm}^{2}\right)$ & $\mathrm{e}(\mathrm{mm})$ & $\rho_{\mathrm{B}}\left(\mathrm{kg} \cdot \mathrm{m}^{-3}\right)$ \\
\hline HL11 & Axial & Beech & 4043 & 19,0 & 786 \\
\hline H7T & Tangential & Beech & 4128 & 12,6 & 736 \\
\hline H8T & Tangential & Beech & 4099 & 13,9 & 763 \\
\hline H11R & Radial & Beech & 3956 & 14,1 & 744 \\
\hline H13R & Radial & Beech & 4096 & 15,0 & 733 \\
\hline 3352B & Axial & Spruce & 3821 & 29,0 & 474 \\
\hline ET4 & Tangential & Spruce & 4015 & 9,9 & 541 \\
\hline ET6 & Tangential & Spruce & 4015 & 9,85 & 488 \\
\hline ET8 & Tangential & Spruce & 4015 & 9,9 & 452 \\
\hline ER6 & Radial & Spruce & 3958 & 9,85 & 493 \\
\hline ER7 & Radial & Spruce & 3958 & 9,85 & 513 \\
\hline
\end{tabular}

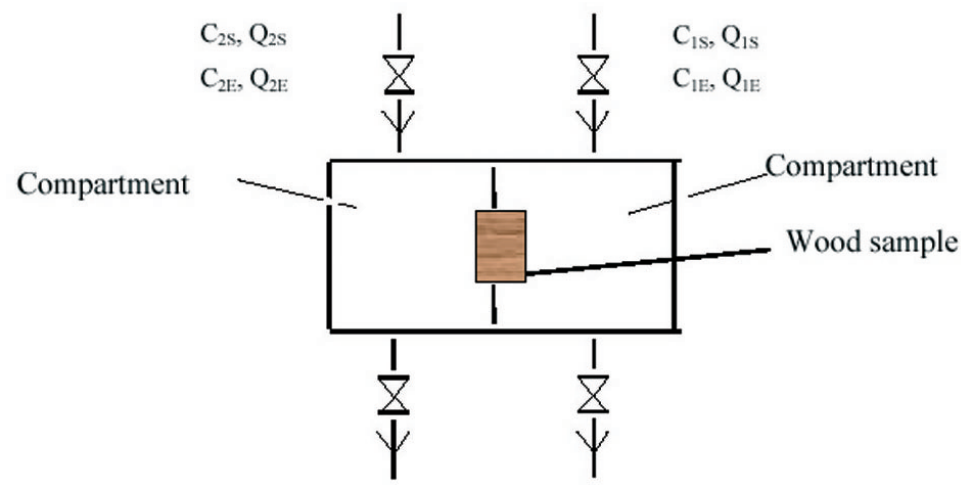

Figure 1: Schema of a Wicke and Kallenbach diffusion cell 
Knowing the apparent diffusivity of the inert solute (DI), the diffusion of water vapor is obtained by writing that the apparent diffusivity is proportional to the real diffusion coefficient, the proportionality coefficient depending only on the geometry and on the structure of the porous medium. Limiting us to the homogeneous model of a porous medium, the apparent diffusivity of the inert solute is linked to the diffusion coefficient of the solute in the gaseous phase by the relation :

$$
\mathrm{D}_{\mathrm{V}}=\frac{\varepsilon}{\tau} D_{\mathrm{I}}
$$

where $\varepsilon$ is the porosity of the porous medium

$\tau$ is the tortuosity factor of the porous medium

$D_{1}$ is the molecular diffusion coefficient of the inert solute (Fick's or Knudsen's diffusion coefficient depending on the mean dimension of pores in the porous medium).

The structural parameters $\varepsilon$ and $\tau$ being independent of the nature of the solute, the apparent diffusivity of water vapor is binding to that of the non- adsorbed solute by the relation :

$$
\mathrm{D}_{\mathrm{V}}=\frac{D_{V}}{D_{I}} \mathrm{D}_{\mathrm{I}}
$$

$D_{\mid}$and $D_{\vee}$ are the diffusion coefficients in the gaseous phase of the inert and of water vapor assessed for geometrical conditions of the porous medium (Fick's diffusion, Knudsen's diffusion, mixed diffusion depending on the pore diameters distribution in the porous medium).

The measurements of bound water diffusivity are done in a vapometer (Perré et al. 1996 ; Perré 1996) simplified in figure 2. It is made of three main elements :

- A container in which a salt-water solution is as far as we know, at a chosen temperature, the vapor pressure of the solute is in thermodynamic equilibrium.

- A water-tight device allowing to limit the solute transfer in the porous medium is placed on the upper part of the container.

- A cell in which the container with the porous sample is placed and in which the temperature (identical to that of the container) and the water vapor concentration of the gaseous atmosphere are controlled.

The water vapor concentrations straight through the wood sample being constant, a stationary water flow appears in the sample. This one is deduced from the mass variation of the container according to time. Knowing the water flux transferred, is equal to the sum of the transferred fluxes in the form of water vapor and bound water and the water vapor diffusivity, it is then possible to estimate the apparent diffusivity of bound water and the water fluxes transferred in the two forms. 


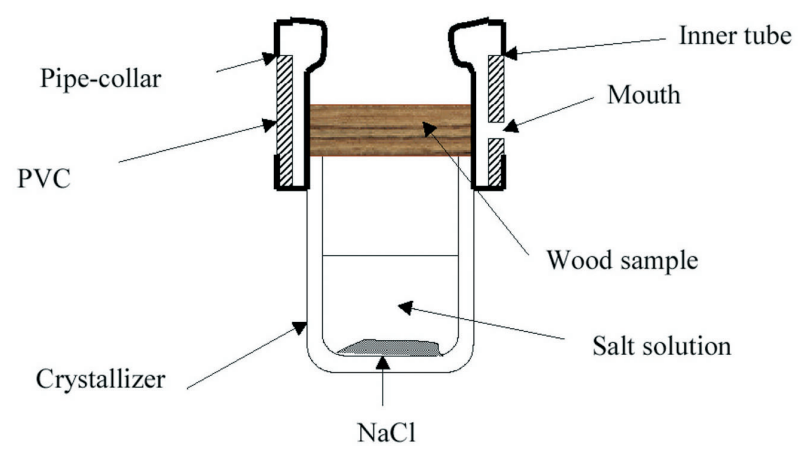

Figure 2: Vapometer's schema

\section{RESULTS AND DISCUSSION}

The geometrical characteristics and the anhydrous density $\rho_{B}$ of wood samples are given in table 1 . In open system, the modelisation of the diffusion cell in the presence of a total pressure difference between the two compartments leads to the relation (Mouchot and Zoulalian 2003):

with

$$
-\operatorname{Ln} \mathrm{A}=\frac{\mathrm{e}}{\mathrm{SD}_{\mathrm{I}}}\left(\mathrm{Q}_{\mathrm{IS}}-\mathrm{Q}_{\mathrm{IE}}\right)
$$

$$
\mathrm{A}=\frac{\mathrm{Q}_{1 \mathrm{~S}}\left(\mathrm{C}_{2 \mathrm{~S}}-\mathrm{C}_{1 \mathrm{~S}}\right)+\mathrm{Q}_{1 \mathrm{E}}\left(\mathrm{C}_{1 \mathrm{E}}-\mathrm{C}_{2 \mathrm{~S}}\right)}{\mathrm{Q}_{1 \mathrm{E}}\left(\mathrm{C}_{1 \mathrm{E}}-\mathrm{C}_{1 \mathrm{~S}}\right)}
$$

where $\quad Q_{1 E}$ and $Q_{1 S}$ represent the volumetric flows at the entrance and the exit of compartment 1 . Compartment 1 is supplied by pure helium

$\mathrm{C}_{1 \mathrm{E}}$ and $\mathrm{C}_{1 \mathrm{~S}}$ represent the molar concentrations of helium in the supplying and exit flows in compartment 1

$\mathrm{C}_{2 \mathrm{~S}}$ represents the molar concentration of helium in the exit flow of compartment 2. Compartment 2 is supplied by pure nitrogen

Plotting the first member of the relation (3) versus $Q_{1 S}-Q_{1 E}$, we must obtain a straight line passing through the origin whose slope allows to estimate $\mathrm{D}_{\mathrm{I}}$. For instance, we plotted in figure 3 the results for the sample 3352B and we see that the relation (3) is quite well verified. In batch system, the modelling of the diffusion cell in the absence of a total pressure difference between the two compartments leads to the relation (Mouchot and Zoulalian 2003):

with

$$
-\mathrm{LnB}=\frac{\mathrm{D}_{\mathrm{I}} \mathrm{S}}{\mathrm{e}} \frac{\left(\mathrm{V}_{1}+\mathrm{V}_{2}\right)}{\mathrm{V}_{1} \mathrm{~V}_{2}} \mathrm{t}
$$

$$
\mathrm{B}=\frac{\left(\mathrm{V}_{1}+\mathrm{V}_{2}\right) \mathrm{C}_{1}-\mathrm{C}_{10} \mathrm{~V}_{1}-\mathrm{C}_{20} \mathrm{~V}_{2}}{\mathrm{~V}_{2}\left(\mathrm{C}_{10}-\mathrm{C}_{20}\right)}
$$

where $\mathrm{V}_{1}$ and $\mathrm{V}_{2}$ are the volumes of compartments 1 and 2

$\mathrm{C}_{1}$ refers to the molar concentration of helium in compartment 1 at instant $\mathrm{t}$

$\mathrm{C}_{10}$ and $\mathrm{C}_{20}$ are the molar concentrations of helium respectively in compartments 1 and 2 at initial instant. 


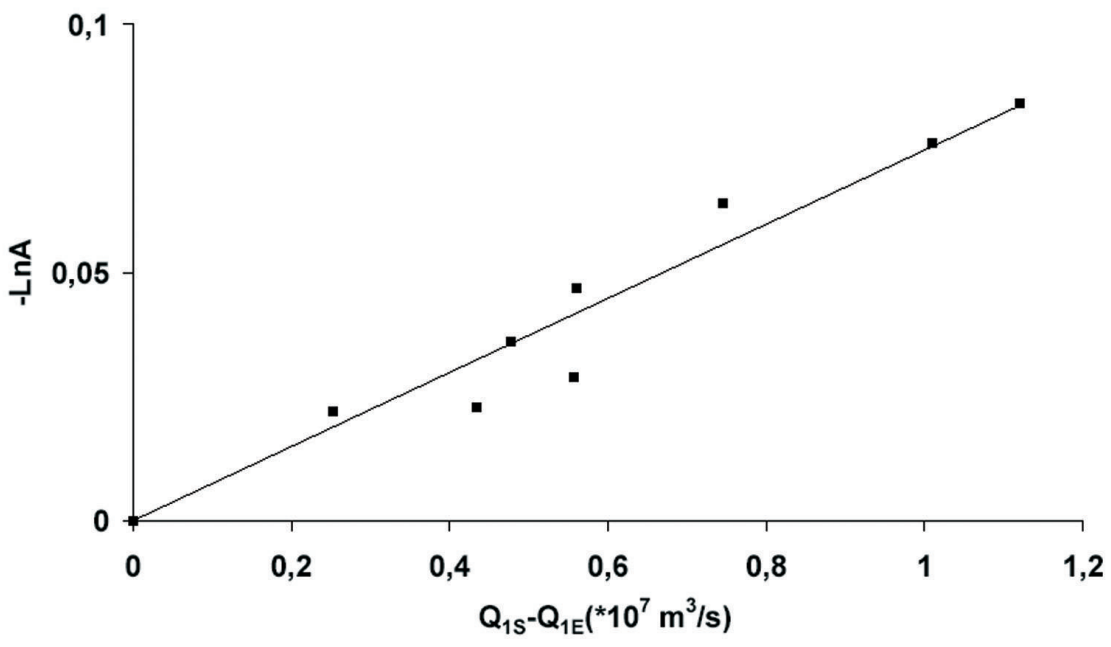

Figure 3: Variation of $-\operatorname{Ln}\left[\frac{Q_{1 S}\left(C_{2 S}-C_{1 S}\right)+Q_{1 E}\left(C_{1 E}-C_{2 S}\right)}{Q_{1 E}\left(C_{1 E}-C_{1 S}\right)}\right]$ versus $Q_{1 S}-Q_{1 E}$ for the sample 3352B

Plotting the first member of the relation (4) versus time, we must obtain a straight line passing through the origin whose slope allows to estimate $\mathrm{D}_{\mathrm{I}}$. For instance, we plotted in figure 4 the results obtained for the sample H13R and we see that the relation (4) is quite well verified. We point out that each point of figures 3 and 4 corresponds to an independent experiment.

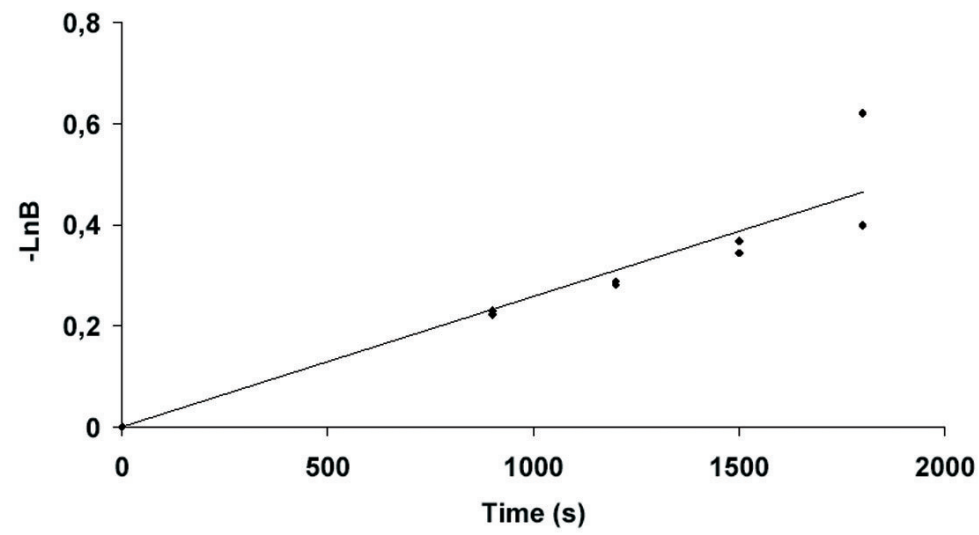

Figure 4: Variation of $-\operatorname{Ln}\left[\frac{\left(V_{1}+V_{2}\right) C_{1}-C_{10} V_{1}-C_{20} V_{2}}{V_{2}\left(C_{10}-C_{20}\right)}\right]$ versus time for the sample

H13R

The previous measurements permit to reach the values of the diffusivities for the eleven samples studied. From these values, we can deduce the diffusivities in relation to the water vapor applying the relation (2). At $30 \mathrm{oC}$, the ratio $D$ $/ D_{\text {hel }}$ evaluated with the help of the Fuller et al. relation, cited in the book of Reid et al.1997, is equal to 0,367. The values of the apparent diffusivities for helium and for water vapor for all the samples studied are given in table 2 . 
Table 2: Values of helium and water vapor apparent diffusivities

\begin{tabular}{|c|c|c|}
\hline Samples & $\mathrm{D}_{\mathrm{I}}\left(\mathrm{m}^{2} / \mathrm{s}\right)$ & $\mathrm{D}_{\mathrm{V}}\left(\mathrm{m}^{2} / \mathrm{s}\right)$ \\
\hline HL11 & $(9,1 \pm 0,4) \cdot 10^{-6}$ & $(3,3 \pm 0,1) \cdot 10^{-6}$ \\
\hline H7T & $(10,0 \pm 2,4) \cdot 10^{-8}$ & $(3,7 \pm 0,9) \cdot 10^{-8}$ \\
\hline H8T & $(10,0 \pm 2,4) \cdot 10^{-8}$ & $(3,7 \pm 0,9) \cdot 10^{-8}$ \\
\hline H11R & $(10,8 \pm 2,2) \cdot 10^{-8}$ & $(4,0 \pm 0,8) \cdot 10^{-8}$ \\
\hline H13R & $(10,8 \pm 2,2) \cdot 10^{-8}$ & $(4,0 \pm 0,8) \cdot 10^{-8}$ \\
\hline 3352B & $(10,1 \pm 0,8) \cdot 10^{-6}$ & $(3,7 \pm 0,3) \cdot 10^{-6}$ \\
\hline ET4 & $(4,6 \pm 1,6) \cdot 10^{-8}$ & $(1,7 \pm 0,6) \cdot 10^{-8}$ \\
\hline ET6 & $(4,6 \pm 1,6) \cdot 10^{-8}$ & $(1,7 \pm 0,6) \cdot 10^{-8}$ \\
\hline ET8 & $(4,6 \pm 1,6) \cdot 10^{-8}$ & $(1,7 \pm 0,6) \cdot 10^{-8}$ \\
\hline ER6 & $(3,5 \pm 1,4) \cdot 10^{-8}$ & $(1,3 \pm 0,5) \cdot 10^{-8}$ \\
\hline ER7 & $(3,5 \pm 1,4) \cdot 10^{-8}$ & $(1,3 \pm 0,5) \cdot 10^{-8}$ \\
\hline
\end{tabular}

For the two species, the diffusivities in the axial direction are clearly greater than the diffusivities in the tangential and radial directions, the latter being however not much different. The axial diffusivities of the two species are close but the tangential and radial diffusivities of beech are twice or three times greater than those of spruce. Then the differences of morphology appear rather on the values of the tangential and radial diffusivities. In the axial direction, the geometrical dimensions of the vessels and lumens of the fibers are such that we are dealing with a Fick diffusive transfer in an ensemble of open pores. Taking the value of the Fick's diffusion coefficient of the binary system water vapor-nitrogen at $30^{\circ} \mathrm{C}$ into account, let $D_{\text {water-nitrogen }}=27.10^{-6} \mathrm{~m} 2 / \mathrm{s}$ and the values of the mean porosity of the beech sample HL11 $(\varepsilon=0,476)$ and spruce sample 3352B $(\varepsilon=0,624)$, the relation (1) permits to estimate a tortuosity factor. We find :

For the sample HL11: $\tau=3,9$

For the sample 3352B : $\tau=5,0$

The values found for the tortuosity factor are similar with those observed for the porous catalyst pellets. The values of the diffusivities in the tangential and radial directions are much weaker because the gaseous solute must go through the cellular walls and follow the rays that are less permeable.

The modelling of the water transfer in the sample in the steady-state leads to the following expression for the transferred mass flow (Mouchot and Zoulalian 2003):

$$
\Phi=\frac{\mathrm{D}_{\mathrm{V}}}{\mathrm{e}}\left(\rho_{1}-\rho_{2}\right) \mathrm{S}+\frac{\mathrm{D}_{\mathrm{A}}}{\mathrm{e}}\left(\mathrm{x}_{1}-\mathrm{x}_{2}\right) \rho_{\mathrm{B}}
$$

where $\rho_{1}$ and $\rho_{2}$ represent the volumetric mass of the vapor water straight through the sample and $x_{1}$ and $\mathrm{x}_{2}$ the absolute moisture of wood in equilibrium with the volumetric mass of water vapor in gas phase $\rho_{1}$ and $\rho_{2}$.

The experiments are done at $35^{\circ} \mathrm{C}$ in conditions where the relative air moisture in the container is about $75 \%$ and that of the cell is about $40 \%$. In these conditions, the values of water vapor volumetric mass $\rho_{1}$ and $\rho_{2}$ and the absolute moistures $x_{1}$ and $x_{2}$ are respectively equal to :

$$
\begin{aligned}
& \rho_{1}=2,904 \cdot 10^{-2} \mathrm{~kg} / \mathrm{m}^{3} \\
& \rho_{2}=1,549 \cdot 10^{-2} \mathrm{~kg} / \mathrm{m}^{3} \\
& \mathrm{x}_{1}=0,140 \text { et } \mathrm{x}_{2}=0,073
\end{aligned}
$$


In the relation (5), the first term represents the water mass flux transferred in the gaseous phase and the second term the water mass flux transferred in the adsorbed phase. The total water mass flux transferred in the sample is obtained with the variation of the container mass carrying the sample In the steadystate, this mass variation must be linear, that is well verified, for instance in the figure 5 for the beech sample HL11. Similar results are obtained for all samples.

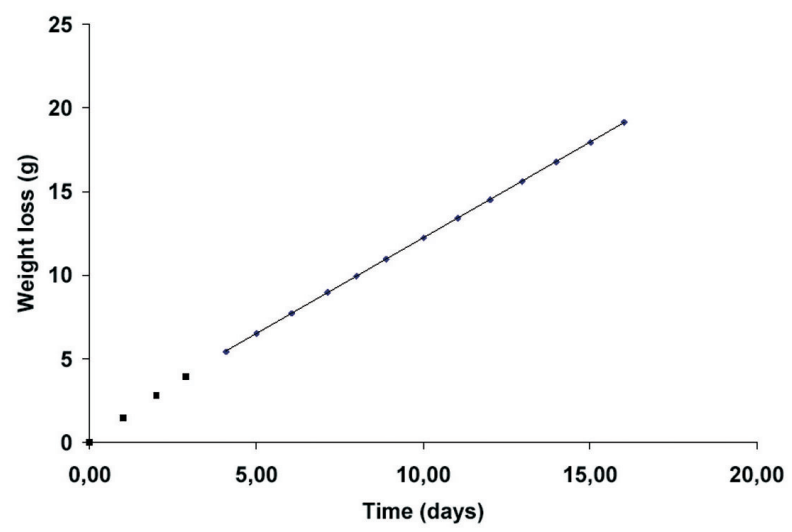

Figure 5: Variation of the weight loss versus time for the sample of beech HL11

Knowing $\Phi(=-\mathrm{dm} / \mathrm{dt}), \rho_{1}, \rho_{2}, \mathrm{x}_{1}, \mathrm{x}_{2}, \mathrm{~S}$, e and $\mathrm{D}_{\mathrm{v}}$, the relation (5) permits to evaluate the diffusivity of the bound water $\mathrm{D}_{\mathrm{A}}$ and to quantify the water fluxes transferred in the sample in the gaseous and adsorbed forms. The results obtained are listed in table 3 .

Table 3: Diffusivities and water transfers given for measurements in vapometer

\begin{tabular}{|c|c|c|c|c|c|c|}
\hline Samples & $\Phi(\mathrm{kg} / \mathrm{s})$ & $\mathrm{D}_{\mathrm{V}}\left(\mathrm{m}^{2} / \mathrm{s}\right)$ & $\mathrm{D}_{\mathrm{A}}\left(\mathrm{m}^{2} / \mathrm{s}\right)$ & $\Phi_{\mathrm{V}}(\mathrm{kg} / \mathrm{s})$ & $\Phi_{\mathrm{A}}(\mathrm{kg} / \mathrm{s})$ & $\Phi_{\mathrm{V}} / \Phi$ \\
\hline HL11 & $1,33.10^{-8}$ & $3,3.10^{-6}$ & $3,38.10^{-10}$ & $0,952 \cdot 10^{-8}$ & $0,378.10^{-8}$ & 0,716 \\
\hline H7T & $4,29 \cdot 10^{-10}$ & $3,7.10^{-8}$ & $1,64.10^{-11}$ & $1,643.10^{-10}$ & $2,647.10^{-10}$ & 0,383 \\
\hline H8T & $5,87.10^{-10}$ & $3,7.10^{-8}$ & $2,91.10^{-11}$ & $1,479.10^{-10}$ & $4,391.10^{-10}$ & 0,252 \\
\hline H11R & $1,03 \cdot 10^{-9}$ & $4,0.10^{-8}$ & $6,25 \cdot 10^{-11}$ & $1,524.10^{-10}$ & $8,776.10^{-10}$ & 0,148 \\
\hline H13R & $1,26.10^{-9}$ & $4,0.10^{-8}$ & $8,27.10^{-11}$ & $1,411.10^{-10}$ & $1,119.10^{-9}$ & 0,112 \\
\hline 3352B & $1,71.10^{-8}$ & $3,7.10^{-6}$ & $2,52 \cdot 10^{-9}$ & $0,660.10^{-8}$ & $1,050.10^{-8}$ & 0,386 \\
\hline ET4 & $1,84.10^{-9}$ & $1,7.10^{-8}$ & $1,19.10^{-10}$ & $0,937 \cdot 10^{-10}$ & $1,746.10^{-9}$ & 0,051 \\
\hline ET6 & $1,42.10^{-9}$ & $1,7.10^{-8}$ & $1,00.10^{-10}$ & $0,937 \cdot 10^{-10}$ & $1,326.10^{-9}$ & 0,066 \\
\hline ET8 & $1,51.10^{-9}$ & $1,7.10^{-8}$ & $1,15 \cdot 10^{-10}$ & $0,936 \cdot 10^{-10}$ & $1,416.10^{-9}$ & 0,062 \\
\hline ER6 & $2,03 \cdot 10^{-9}$ & $1,3.10^{-8}$ & $1,48.10^{-10}$ & $0,710 \cdot 10^{-10}$ & $1,955.10^{-9}$ & 0,035 \\
\hline ER7 & $1,74 \cdot 10^{-9}$ & $1,3 \cdot 10^{-8}$ & $1,23 \cdot 10^{-10}$ & $0,696 \cdot 10^{-10}$ & $1,670.10^{-9}$ & 0,040 \\
\hline
\end{tabular}

The diffusivity values for bound water are lower than those for water vapor, that is logical insofar as such that the physical state of the bound water is closer to the liquid state than to the gaseous state. The diffusivities in the axial direction are greater than those in the tangential and radial directions but the ratio between the axial and the tangential or radial diffusivities is lower than the ratio observed for the diffusivities in the gaseous phase. We can notice that the diffusivities in the tangential direction are lower than those in the radial direction. The presence of the rays is probably at the origin of the increase of the radial diffusivity compared to the tangential diffusivity.

The results were more interesting concerning the water fluxes transferred. For beech in the axial direction, the water flux is mainly transferred in the gaseous phase. In the tangential and radial directions, the percent of water fluxes transferred in the gaseous phase are lower $30 \%$ in the tangential direction and between 10 and $15 \%$ in the radial direction), but they are not negligible. For spruce, the water flow 
transferred in the gaseous phase remains high in the axial direction. On the other hand, in the tangential and radial directions, they are almost negligible. In these conditions, the diffusive transport of water is mainly done in the adsorbed form.

It can also be noticed that the diffusivities of the bound water for spruce are, whatever the considered direction may be greater than the diffusivities of the bound water observed for beech samples. The moisture of the two species being identical (same ratio water mass/mass of dry wood), these differences are allocated to the morphology's difference of the two species, but at this stage it is difficult to suggest a scientific explication taking into account the observed variations. It will be necessary to measure again the bound water diffusivities of several wood species in order to verify a possible correlation between the wood anatomy and the values of water diffusivities in gaseous and adsorbed phases.

The measurements of the transfer in the vapometer can be used too to evaluate a global apparent diffusivity of water, not distinguishing the water vapor and bound water.

Two diffusivities can be deduced depending on whether driving force is considered $\rho_{1}-\rho_{2}$ or $x_{1}-x_{2}$ . They are defined by the following relations :

$$
\Phi=\frac{D^{*} S}{\mathrm{e}}\left(\rho_{1}-\rho_{2}\right)=\frac{\mathrm{D}^{* *} \rho_{\mathrm{B}}}{\mathrm{e}}\left(\mathrm{x}_{1}-\mathrm{x}_{2}\right)
$$

The values of the diffusivities $\mathrm{D}^{*}$ and $\mathrm{D}^{* *}$ for all samples studied are given in the table 4 . $\mathrm{D}^{*}$ and $\mathrm{D}^{* *}$ can be compared to $\mathrm{D}_{\mathrm{V}}$ and $\mathrm{D}_{\mathrm{A}}$ respectively, but the effect of the morphology is reduced and these diffusivities do not allow the identification of what the percentage of the transferred flux in gaseous phase and adsorbed phase is.

Table 4: Values of diffusivities D* and D* deduced from measurements in vapometer and equations (6)

\begin{tabular}{|c|c|c|c|c|}
\hline Samples & $\mathrm{D}_{\mathrm{V}}\left(\mathrm{m}^{2} / \mathrm{s}\right)$ & $\mathrm{D}^{*}\left(\mathrm{~m}^{2} / \mathrm{s}\right)$ & $\mathrm{D}_{\mathrm{A}}\left(\mathrm{m}^{2} / \mathrm{s}\right)$ & $\mathrm{D}^{* *}\left(\mathrm{~m}^{2} / \mathrm{s}\right)$ \\
\hline HL11 & $3,3.10^{-6}$ & $4,61.10^{-6}$ & $3,38.10^{-10}$ & $1,19.10^{-9}$ \\
\hline H7T & $3,7.10^{-8}$ & $9,67.10^{-8}$ & $1,64.10^{-11}$ & $2,66.10^{-11}$ \\
\hline H8T & $3,7.10^{-8}$ & $1,47.10^{-7}$ & $2,91.10^{-11}$ & $3,89.10^{-11}$ \\
\hline H11R & $4,0.10^{-8}$ & $2,70.10^{-7}$ & $6,25.10^{-11}$ & $7,34.10^{-11}$ \\
\hline H13R & $4,0.10^{-8}$ & $3,40.10^{-7}$ & $8,27.10^{-11}$ & $9,37.10^{-11}$ \\
\hline 3352B & $3,7.10^{-6}$ & $9,58.10^{-6}$ & $2,52.10^{-9}$ & $4,10.10^{-9}$ \\
\hline ET4 & $1,7.10^{-8}$ & $3,34.10^{-7}$ & $1,19.10^{-10}$ & $1,25.10^{-10}$ \\
\hline ET6 & $1,7.10^{-8}$ & $2,57.10^{-7}$ & $1,00.10^{-10}$ & $1,07.10^{-10}$ \\
\hline ET8 & $1,7.10^{-8}$ & $2,75.10^{-7}$ & $1,15.10^{-10}$ & $1,23.10^{-10}$ \\
\hline ER6 & $1,3.10^{-8}$ & $3,74.10^{-7}$ & $1,48.10^{-10}$ & $1,53.10^{-10}$ \\
\hline ER7 & $1,3.10^{-8}$ & $3,23.10^{-7}$ & $1,23.10^{-10}$ & $1,28.10^{-10}$ \\
\hline
\end{tabular}

Finally, we have shown that the use of the value of the diffusivity $\mathrm{D}^{* *}$ does not provide the mass variations of the samples during transitive experiments of adsorption or desorption. The observed differences are probably due to the non thermodynamic equilibrium between the water vapor and the bound water.

We report however that the values of $\mathrm{D}^{*}$ and $\mathrm{D}^{* *}$ are quite compatible with the values reported in the literature using the experimental device of the vapometer (Perré et al 1996, Perré 1996, Choong 1965, Siau and Babiak 1983). 


\section{CONCLUSIONS}

For beech and spruces species, we have determined the diffusivities of water vapor and bound water in the three orthotropic directions of wood and specified for each direction the transfer part in gaseous phase and in adsorbed phase. For beech, the transferred flux in the gaseous phase is predominant in the axial direction and it exists in the tangential and radial directions. For spruce, the transferred flux in the gaseous phase represents $40 \%$ of the total flux in the axial direction, but in the radial and tangential directions, water is transferred mainly in the adsorbed form.

The morphology of the species plays an important role on the values of the diffusivities in gaseous phase and adsorbed phase, but its influence on the diffusivities in gaseous phase could be very different and even opposite to the diffusivities observed for the bound water.

Finally, to better determine the probable relations between the morphology of the species and the values of the diffusivities in gaseous and adsorbed phase, it is necessary to carry out new experiments on species whose morphologies are different from those of the two studied species. For the diffusivities of water in the adsorbed phase, it is also necessary to plan some measurements in variable wood moisture range, in order to specify the possible variation of the diffusivities versus wood moisture.

\section{LITERATURE CITED}

Choong, E.T. 1965. Diffusion coefficients of softwoods by steady-state and theoretical methods. Forest Prod. J., 15(1):21-27.

Mouchot, N.; Zoulalian, A. 2003. Détermination des caractéristiques diffusionnelles de transfert de masse d'un soluté gazeux adsorbable dans un solide poreux. In $6^{\text {ème }}$ Colloque Interuniversitaire FrancoQuébécois Thermique des Systèmes, Québec.

Perré, P.; Zohoun, S.; Brandão, A. 1996. Détermination de la diffusivité massique en régime permanent: résultats d'une campagne de mesures sur différentes essences tempérées et tropicales, Sciences et Industries du Bois - $4^{\text {ème }}$ colloque, 11-12-13 Sept. 1996, Nancy, ARBOLOR.

Perré, P. 1996. Mesure en régime permanent de la diffusivité massique dans le domaine hygroscopiqueÊ: présentation du système PVC - CHA, Cahiers d'ARBOLOR.

Reid, R.C.; Prausnitz, J.M.; Sherwood, T.K. 1977. "Chapter 11 : diffusion coefficients" in The properties of gases and liquids-Third edition, The edition McGraw-Hill, pp. 544-601.

Siau, J.F.; Babiak, M. 1983. Experiments on nonisothermal moisture movement in wood. Wood and Fiber Sci., 15(1):40-46. 
